\title{
Countable Fréchet Boolean groups: An independence result
}

\author{
Jörg Brendle* \\ Graduate School of Engineering \\ Kobe University \\ Rokko-dai 1-1, Nada-ku \\ Kobe 657-8501, Japan \\ email: brendle@kurt.scitec.kobe-u.ac.jp \\ and \\ Michael Hrušák ${ }^{\dagger}$ \\ Instituto de Matemáticas \\ Universidad Nacional Autónoma de México (Morelia) \\ A.P. 61-3 Xangari \\ Morelia, Michoacán 58089, México \\ email: michael@matmor.unam.mx
}

November 9, 2008

\begin{abstract}
It is relatively consistent with ZFC that every countable $F U_{\text {fin }}$ space of weight $\aleph_{1}$ is metrizable. This provides a partial answer to a question of G. Gruenhage and P. Szeptycki [GS1].
\end{abstract}

\section{Introduction}

Classical metrization theorem of Birkhoff and Kakutani states that a $T_{1}$ topological group is metrizable if and only if it is first countable. The results contained here are motivated by the following question:

Question 1. (V.I. Malykhin)[Ar, MT] Is there a countable Fréchet-Urysohn topological group that is not metrizable?

*Partially supported by Grant-in-Aid for Scientific Research (C) 17540116 and (C) 19540127, Japan Society for the Promotion of Science.

${ }^{\dagger}$ Partially supported by PAPIIT grant IN101608 and CONACYT grant 46337. 
Recall that a topological space $X$ is Fréchet-Urysohn (or just Fréchet) if whenever a point $x \in X$ is in the closure of a set $A \subseteq X$, there is a sequence of elements of $A$ converging to $x$. In fact, the question can be reformulated by asking for the existence of a separable Fréchet-Urysohn topological group that is not metrizable. On the other hand there are non-separable, even $\sigma$-compact examples, for instance the direct sum of uncountably many copies of the circle.

It is well known (see [Ny1, Ny2, GN]) that the answer to Malykhin's question is consistently positive, i.e. under either of the following assumptions: $\mathfrak{p}>\omega_{1}, \mathfrak{b}=\mathfrak{p}$ and the existence of an uncountable $\gamma$-set. In fact, either of the assumptions implies the existence of a simply described non-metrizable Fréchet-Urysohn group topology on the Boolean group $G=\left([\omega]^{<\omega}, \triangle\right)$ of finite subsets of $\omega$ with symmetric difference as the group operation.

Let $\mathcal{F}$ be a free filter on $\omega$. Then

$$
\mathcal{F}^{<\omega}:=\left\{A \subseteq[\omega]^{<\omega}: \text { there is } F \in \mathcal{F} \text { such that }[F]^{<\omega} \subseteq A\right\}
$$

is a filter on $[\omega]^{<\omega}$. By stipulating that $\mathcal{F}^{<\omega}$ is a neighbourhood base at $\emptyset$, we introduce a group topology $\tau_{\mathcal{F}}$ on $G$. Note that, by definition, $\mathcal{F}^{<\omega}$ is generated by sets of the form $[F]^{<\omega}, F \in \mathcal{F}$. Also, any set of the form $[F]^{<\omega}$ is a subgroup of $G$. So the $[F]^{<\omega}, F \in \mathcal{F}$, are in fact open subgroups which generate the neighbourhood base at $\emptyset$.

Observation 1. The following are equivalent:

1. $\left(G, \tau_{\mathcal{F}}\right)$ is first-countable (equivalently, metrizable);

2. $\mathcal{F}^{<\omega}$ is countably generated;

3. $\mathcal{F}$ is countably generated.

A filter $\mathcal{F}$ such that the topological group $\left(G, \tau_{\mathcal{F}}\right)$ is Fréchet is called an $F U_{\text {fin }}$-filter, see [GS1, GS2, RS, Si]. This is conveniently expressed in the dual language of ideals. If $\mathcal{I}=\mathcal{F}^{*}$ is the dual ideal of $\mathcal{F}, \mathcal{I}^{<\omega}:=$ $\left\{A \subseteq[\omega]^{<\omega}\right.$ : for some $I \in \mathcal{I}, a \cap I \neq \emptyset$ for all $\left.a \in A\right\}$ is the dual ideal of $\mathcal{F}^{<\omega}$ and is generated by sets of the form $\left\{a \in[\omega]^{<\omega}: a \cap I \neq \emptyset\right\}, I \in \mathcal{I}$.

Let $\mathcal{I}$ be an ideal on $\omega$. Define the orthogonal ideal $\mathcal{I}^{\perp}:=\{A \subseteq \omega$ : $|I \cap A|<\omega$ for all $I \in \mathcal{I}\}$. Clearly $\mathcal{I}^{\perp \perp} \supseteq \mathcal{I}$ and $\mathcal{I}^{\perp \perp \perp}=\mathcal{I}^{\perp}$. We call $\mathcal{I}$ Fréchet if $\mathcal{I}^{\perp \perp}=\mathcal{I}$. This is equivalent to saying that for all $X \in \mathcal{I}^{+}$ there is $C \in[X]^{\omega}$ such that $C \in \mathcal{I}^{\perp}$. $\mathcal{I}$ is said to be tall if for all $X \in[\omega]^{\omega}$ there is $C \in[X]^{\omega}$ such that $C \in \mathcal{I}$.

Observation 2. 1. Every countably generated ideal is Fréchet.

2. If $\mathcal{I}$ is tall, then $\mathcal{I}$ is not Fréchet.

3. $\mathcal{I}^{\perp}$ is always Fréchet.

The following is a reformulation of a result of [RS]. We include the simple proof for the sake of completeness.

Lemma 1. The topological group $\left(G, \tau_{\mathcal{F}}\right)$ is Fréchet iff the ideal $\mathcal{I}^{<\omega}$ is Fréchet where $\mathcal{I}=\mathcal{F}^{*}$.

Proof. $(\Longrightarrow)$ Let $X \in\left(\mathcal{I}^{<\omega}\right)^{+}$. This means that for all $I \in \mathcal{I}$ there is $a \in X$ with $I \cap a=\emptyset$. Equivalently, for all $F \in \mathcal{F}$ there is $a \in X$ 
with $a \subseteq F$. So $X \cap[F]^{<\omega} \neq \emptyset$ for all $F \in \mathcal{F}$. By definition of $\tau_{\mathcal{F}}, \emptyset$ belongs to the closure $\bar{X}$ of $X$. Since $\tau_{\mathcal{F}}$ is Fréchet, there is a sequence $\left(a_{n}: n \in \omega\right) \subseteq X$ converging to $\emptyset$. Let $A=\left\{a_{n}: n \in \omega\right\}$. Then $A \in\left(\mathcal{I}^{<\omega}\right)^{\perp}$.

$(\Longleftarrow)$ Let $X \subseteq[\omega]^{<\omega}$ be such that $\emptyset \in \bar{X}$. So $X \cap[F]^{<\omega} \neq \emptyset$ for all $F \in \mathcal{F}$. This means that $X \in\left(\mathcal{I}^{<\omega}\right)^{+}$. Since $\mathcal{I}^{<\omega}$ is Fréchet, there is $A \in[X]^{\omega}$ such that $A \in\left(\mathcal{I}^{<\omega}\right)^{\perp}$. Writing $A=\left\{a_{n}: n \in \omega\right\}$ we see that $a_{n}$ converges to $\emptyset$.

G. Gruenhage and P. Szeptycki [GS1] asked whether $F U_{\text {fin }}$-filters of uncountable character exist in ZFC. We shall show:

Theorem 1. It is consistent that $\mathfrak{c}=\aleph_{2}$ and for all filters $\mathcal{F}$, if $\mathcal{F}$ is $\omega_{1}$-generated, then $\left(G, \tau_{\mathcal{F}}\right)$ is not Fréchet.

Or, equivalently:

Theorem 2. It is consistent that $\mathfrak{c}=\aleph_{2}$ and for all ideals $\mathcal{I}$, if $\mathcal{I}$ is $\omega_{1}$-generated, then $\mathcal{I}^{<\omega}$ is not Fréchet.

\section{Combinatorics and forcing}

Assume a filter $\mathcal{F}$ on $\omega$ and its dual ideal $\mathcal{I}=\mathcal{F}^{*}$ are given. For $a \in[\omega]^{<\omega}$ define cone $(a)=\left\{b \in[\omega]^{<\omega}: a \subseteq b\right\}$, the cone over $a$.

Define the following families on $[\omega]^{<\omega}$ :

$$
\begin{aligned}
\mathcal{G}=\mathcal{G}_{\mathcal{I}} & =\left\{A \subseteq[\omega]^{<\omega}: \forall I \in \mathcal{I} \exists a(a \cap I=\emptyset \wedge \operatorname{cone}(a) \subseteq A)\right\} \\
\mathcal{J}=\mathcal{J}_{\mathcal{I}} & =\left\{A \subseteq[\omega]^{<\omega}: \forall I \in \mathcal{I} \exists a(a \cap I=\emptyset \wedge \operatorname{cone}(a) \cap A=\emptyset)\right\} \\
\mathcal{J}^{+} & =\left\{A \subseteq[\omega]^{<\omega}: \exists I \in \mathcal{I} \forall a(a \cap I=\emptyset \rightarrow \operatorname{cone}(a) \cap A \neq \emptyset)\right\}
\end{aligned}
$$

Lemma 2. $\mathcal{G}$ is a filter, $\mathcal{J}=\mathcal{G}^{*}$ is the dual ideal, and $\mathcal{J}^{+}$is the collection of $\mathcal{J}$-positive sets. Furthermore, $\mathcal{J}$ is a Fréchet ideal.

Proof. Only the last assertion needs proof. Let $A \in \mathcal{J}^{+}$and let $I$ witness this. Let $\left\{k_{n}: n \in \omega\right\}$ enumerate $\omega \backslash I$, and set $a_{n}=\left\{k_{i}: i<n\right\}$. Then for each $n$ there is $b_{n} \supseteq a_{n}$ with $b_{n} \in A$. Clearly $B=\left\{b_{n}: n \in \omega\right\} \in \mathcal{J}^{\perp}$.

We consider $\mathbb{L}(\mathcal{H})$, Laver forcing associated to a filter $\mathcal{H}$ on a countable set $W$. It is defined as the set of those trees $T \subseteq W^{<\omega}$ for which there is an $s \in T$ (called the stem of $T$ ) such that for all $t \in T, t \subseteq s$ or $s \subseteq t$ and such that for all $t \in T$ with $t \supseteq s$ the set $\operatorname{succ}_{T}(t)=\left\{a \in W: t^{\frown} a \in T\right\}$ belongs to $\mathcal{H}$. It is ordered by inclusion. The forcing $\mathbb{L}(\mathcal{H})$ is $\sigma$-centered and generically adds a function $\dot{\ell}: \omega \rightarrow W$.

In what follows, we denote by $\dot{X}=\operatorname{ran}(\dot{\ell})$ the $\mathbb{L}(\mathcal{G})$-name for the generic subset of $[\omega]^{<\omega}$ added by $\mathbb{L}(\mathcal{G})$, where $\mathcal{G}$ is the filter defined above.

Lemma 3. $\Vdash_{\mathbb{L}(\mathcal{G})} \dot{X} \in\left(\dot{\mathcal{I}}^{<\omega}\right)^{+}$

Proof. This is a straightforward genericity argument. 
We next intend to prove that if $\mathcal{I}$ is not countably generated, then $\mathbb{L}(\mathcal{G})$ forces that $\dot{X} \in\left(\dot{\mathcal{I}}^{<\omega}\right)^{\perp \perp}$ (Lemma 4 below). For preservation purposes (see Proposition 1 and Lemma 5), however, we need a somewhat stronger result. First, some additional notions.

We say that a family $\mathcal{K} \subseteq[\omega]^{\omega}$ is countably tall (or $\omega$-hitting) [Do] if given $\left(A_{n}: n \in \omega\right) \subseteq[\omega]^{\omega}$ there is an $I \in \mathcal{K}$ such that $I \cap A_{n}$ is infinite for all $n$. Clearly, every countably tall ideal is tall. An important property of countably tall families, which will be used several times in what follows, is that if a countably tall family is partitioned into countably many pieces, then at least one of the pieces is countably tall. For an ideal $\mathcal{K}$ and $X \in \mathcal{K}^{+}$, define the restriction ideal of subsets of $X$ by $\mathcal{K} \uparrow X=\{I \cap X: I \in \mathcal{K}\}$.

Observation 3. Assume $\mathcal{K}\left\lceil X\right.$ is tall. Then $X \in \mathcal{K}^{\perp \perp}$.

Lemma 4. Assume $\mathcal{I}$ is not countably generated. Then $\Vdash_{\mathbb{L}(\mathcal{G})}$ " $\mathcal{I}<\omega \mid \dot{X}$ is countably tall'. In particular, $\Vdash_{\mathbb{L}(\mathcal{G})} \dot{X} \in\left(\dot{\mathcal{I}}^{<\omega}\right)^{\perp \perp}$.

Proof. Let $\left(\dot{A}_{n}: n \in \omega\right)$ be a sequence of names for infinite subsets of $[\omega]^{<\omega}$. We may suppose the $\dot{A}_{n}$ are forced to be subsets of $\dot{X}$. Assume, by way of contradiction, that for all $I \in \mathcal{I}$ there are $p_{I} \in \mathbb{L}(\mathcal{G})$, and natural numbers $n_{I}, m_{I}$ such that

$$
p_{I} \Vdash \bigcup \dot{A}_{n_{I}} \cap I \subseteq m_{I} .
$$

Recall the standard rank analysis for Laver forcing [Br1][Br2]. For $s \in\left([\omega]^{<\omega}\right)^{<\omega}$, say $s$ favors $a \in \dot{A}_{n}$ if there is no condition $p \in \mathbb{L}(\mathcal{G})$ with stem $s$ such that $p \Vdash a \notin \dot{A}_{n}$, or, equivalently, every condition $p \in \mathbb{L}(\mathcal{G})$ with stem $s$ has an extension $q$ such that $q \Vdash a \in \dot{A}_{n}$. Define the rank $\operatorname{rk}_{n}(s)$ by recursion on the ordinals by

$$
\begin{aligned}
& \operatorname{rk}_{n}(s)=0 \quad \text { iff } \quad \exists B \in \mathcal{G}^{+} \forall b \in B\left(s \frown b \text { favors } b \in \dot{A}_{n}\right) \\
& \operatorname{rk}_{n}(s) \leq \alpha \quad \text { iff } \quad \exists B \in \mathcal{G}^{+} \forall b \in B\left(\operatorname{rk}_{n}(s \frown b)<\alpha\right)
\end{aligned}
$$

for $\alpha>0$

Claim 1. $\operatorname{rk}_{n}(s)<\infty$ for all $s$ and $n$.

Proof. Fix $n$. Let $k \in \omega$. Define an auxiliary rank $\rho_{k}(s)$ by recursion such that

$$
\rho_{k}(s)=0 \text { iff } \exists b \nsubseteq k\left(s \text { favors } b \in \dot{A}_{n}\right)
$$

and $\rho_{k}(s) \leq \alpha$ is defined as for $\mathrm{rk}_{n}$, for $\alpha>0$. As $\dot{A}_{n}$ is forced to be infinite, it is straightforward to see that $\rho_{k}(s)<\infty$ for all $s$ and $k$. Also note that since $\dot{A}_{n}$ is forced to be a subset of the generic $\dot{X}$, any $s$ can favor only elements of $\operatorname{ran}(s)$.

If $\rho_{k}(s)=1$, then there is a $\mathcal{G}$-positive set of $b$ such that $s \frown b$ favors $c \in \dot{A}_{n}$ for some $c=c_{b}$ with $c \nsubseteq k$. If on a $\mathcal{G}$-positive set, the same $c$ works, we get $\rho_{k}(s)=0$, a contradiction. Since $c_{b} \in \operatorname{ran}(s) \cup\{b\}$, it follows that on a $\mathcal{G}$-positive set, $c_{b}=b$. This, however, means that $\operatorname{rk}_{n}(s)=0$.

Now, let $k>\max (\bigcup \operatorname{ran}(s))$. Then $\rho_{k}(s) \geq 1$. By the preceding paragraph and induction, we see that $\operatorname{rk}_{n}(s)<\infty$, as required. 
We continue with the proof of the lemma. Let $s_{I}$ be the stem of $p_{I}$. By strengthening the $p_{I}$, if necessary, we may assume that $\operatorname{rk}_{n_{I}}\left(s_{I}\right)=0$ for all $I$.

Since the ideal $\mathcal{I}$ is not countably generated, there are $s$ and $n$ such that for no $J \in \mathcal{I}$, we have that for all $I$ with $s_{I}=s$ and $n_{I}=n$ do we have $I \subseteq^{*} J$. Fix such $s$ and $n$.

Let $B \in \mathcal{G}^{+}$witness that $\operatorname{rk}_{n}(s)=0$. Let $I_{0} \in \mathcal{I}$ witness that $B \in \mathcal{G}^{+}$. Recall that this means that for all $a \in[\omega]^{<\omega}$ with $a \cap I_{0}=\emptyset$, we have cone $(a) \cap B \neq \emptyset$.

Find $I \in \mathcal{I}$ such that $s_{I}=s, n_{I}=n$, and $I \backslash I_{0}$ is infinite. By definition of $\mathcal{G}$, there is $a$ with $a \cap I_{0}=\emptyset$ such that cone $(a) \subseteq \operatorname{succ}_{p_{I}}(s)$. Since $I \backslash I_{0}$ is infinite, we may assume that $(a \cap I) \backslash m_{I} \neq \emptyset$. Find $b \in \operatorname{cone}(a) \cap B$. So $b \in B \cap \operatorname{succ}_{p_{I}}(s)$, and $s \frown b$ favors $b \in \dot{A}_{n}$ by definition of $B$. Thus we can construct a condition $q \leq p_{I}$ whose stem extends $s \frown b$ such that $q \Vdash b \in \dot{A}_{n}$. Since $(b \cap I) \backslash m_{I} \neq \emptyset$, this is a contradiction to the initial assumption $(\star)$. Thus, for some $I \in \mathcal{I}$,

$$
\Vdash \bigcup \dot{A}_{n} \cap I \text { is infinite for all } n \text {. }
$$

This immediately implies countable tallness of the restriction ideal in the generic extension.

We now turn to the preservation of countable tallness in iterations. In order to do that we introduce a stronger property: We say that a forcing notion $\mathbb{P}$ strongly preserves countable tallness if for every sequence $\left(\dot{A}_{n}: n \in \omega\right)$ of $\mathbb{P}$-names for infinite subsets of $\omega$ there is a sequence $\left(B_{n}: n \in \omega\right)$ of infinite subsets of $\omega$ such that for any $B \in[\omega]^{\omega}$, if $B \cap B_{n}$ is infinite for all $n$ then $\vdash_{\mathbb{P}} B \cap \dot{A}_{n}$ is infinite for all $n$. Recall the definition of Katětov order (see [HG, HZ]): Given two ideals $\mathcal{I}, \mathcal{J}$ on $\omega$, we say that $\mathcal{I} \leq_{K} \mathcal{J}$ if there is a function $f: \omega \rightarrow \omega$ such that $f^{-1}[I] \in \mathcal{J}$ for every $I \in \mathcal{I}$.

Proposition 1. Let $\mathcal{K}$ be an ideal on $\omega$ and let $\mathcal{H}=\mathcal{K}^{*}$ be the dual filter. then the following are equivalent:

1. For every $X \in \mathcal{K}^{+}$and every $\mathcal{J} \leq_{K} \mathcal{K}\lceil X$ the ideal $\mathcal{J}$ is not countably tall.

2. $\mathbb{L}(\mathcal{H})$ strongly preserves countable tallness.

3. $\mathbb{L}(\mathcal{H})$ preserves countable tallness, i.e. if $\mathcal{L}$ is countably tall, then $\Vdash_{\mathbb{L}(\mathcal{H})}$ " $\mathcal{L}$ is countably tall".

Proof. $\left(1 \Rightarrow 2\right.$.) Let $\left(\dot{A}_{n}: n \in \omega\right)$ be names for countable subsets of $\omega$. Aiming towards a contradiction, assume that for each $\left(B_{n}: n \in \omega\right)$ there is a $B \in[\omega]^{\omega}$ such that $B \cap B_{n}$ is infinite for all $n$, yet there are a condition $p_{B}$ and natural numbers $n_{B}, m_{B}$ such that

$$
p_{B} \Vdash B \cap \dot{A}_{n_{B}} \subseteq m_{B} .
$$

Let $\mathcal{B}$ be the family of all such $B \in[\omega]^{\omega}$, i.e., the family of all $B \in[\omega]^{\omega}$ such that there are a condition $p_{B}$ and natural numbers $n_{B}, m_{B}$ such that $p_{B} \Vdash B \cap \dot{A}_{n_{B}} \subseteq m_{B}$. By our assumption $\mathcal{B}$ is countably tall. 
Define a new rank function $\operatorname{rank}_{n}$ (cf. the proof of Lemma 4) by recursion on the ordinals as follows:

$$
\begin{gathered}
\operatorname{rank}_{n}(s)=0 \quad \text { iff } \quad \text { either } \exists Z \in[\omega]^{\omega} \forall k \in Z\left(s \text { favors } k \in \dot{A}_{n}\right) \\
\text { or } \exists X \in \mathcal{H}^{+}, f: X \rightarrow \omega \\
\forall \ell \in X\left(s \frown \ell \text { favors } f(\ell) \in \dot{A}_{n}\right) \\
\text { and } \forall k \in \omega\left(f^{-1}(k) \in \mathcal{K}\right)
\end{gathered}
$$

and $\operatorname{rank}_{n}(s) \leq \alpha$ is defined as for $\operatorname{rk}_{n}$, for $\alpha>0$.

Claim 2. $\operatorname{rank}_{n}(s)<\infty$ for all $s$ and $n$.

Proof. Fix $n$. Assume $\operatorname{rank}_{n}(s)=\infty$. So $Z:=\left\{k: s\right.$ favors $\left.k \in \dot{A}_{n}\right\}$ is finite. Recursively build $p \in \mathbb{L}(\mathcal{H})$ with stem $s$ such that for all $t \in p$ extending $s$,

- $\operatorname{rank}_{n}(t)=\infty$, and

- $\left\{k: t\right.$ favors $\left.k \in \dot{A}_{n}\right\} \subseteq Z$.

Let such $t$ be given. First, there is $X_{0} \in \mathcal{H}$ such that $\operatorname{rank}_{n}(t \frown \ell)=\infty$ for all $\ell \in X_{0}$. Let $X_{1}=\left\{\ell \in X_{0}: \exists k \notin Z\right.$ ( $t \frown \ell$ favors $\left.\left.k \in \dot{A}_{n}\right)\right\}$. If $X_{1} \in \mathcal{H}^{+}$, then we can define a function as in the definition of $\operatorname{rank}_{n}$, and so $\operatorname{rank}_{n}(t)=0$, a contradiction. Thus $X_{1} \in \mathcal{K}$ and $X_{0} \backslash X_{1} \in \mathcal{H}$. For $t \frown \ell$ with $\ell \in X_{0} \backslash X_{1}$, both clauses above are satisfied, and the construction proceeds.

Now find $q \leq p$ and $k \notin Z$ such that $q \Vdash k \in \dot{A}_{n}$. Then the stem of $q$ in particular favors $k \in \dot{A}_{n}$, a contradiction.

We continue with the proof of $(1 \Rightarrow 2)$. Let $s_{B}$ be the stem of $p_{B}$. By strengthening the $p_{B}$, if necessary, we may assume that $\operatorname{rank}_{n_{B}}\left(s_{B}\right)=0$ for all $B \in \mathcal{B}$. Since $\mathcal{B}$ is countably tall, there are $s$ and $n$ such that the family $\mathcal{B}_{0}=\left\{B \in \mathcal{B}: s=s_{B}\right.$ and $\left.n=n_{B}\right\}$ is countably tall. Fix such $s$ and $n$.

We consider two cases, according to the definition of $\operatorname{rank}_{n}$.

Case 1. $\exists Z \in[\omega]^{\omega} \forall k \in Z$ ( $s$ favors $\left.k \in \dot{A}_{n}\right)$.

Let $B \in \mathcal{B}_{0}$ be such that $B \cap Z$ is infinite. So there is $k>m_{B}$ such that $k \in B \cap Z$. Thus there is $q \leq p_{B}$ with $q \Vdash k \in \dot{A}_{n}$, a contradiction.

Case 2. $\exists X \in \mathcal{H}^{+}, f: X \rightarrow \omega \forall \ell \in X(s \frown \ell$ favors $f(\ell) \in$ $\left.\dot{A}_{n}\right)$ and $\forall k \in \omega\left(f^{-1}(k) \in \mathcal{K}\right)$.

As $\mathcal{B}_{0}$ is countably tall, there is a $B \in \mathcal{B}_{0}$ such that $f^{-1}[B] \in \mathcal{K}^{+}$, as otherwise, $f$ witnesses that the ideal generated by $\mathcal{B}_{0}$ is Katětov below $\mathcal{K}\left\lceil X\right.$, which contradicts (1). So there is $k \in B \cap \operatorname{ran}(f), k>m_{B}$, such that $f^{-1}(k) \cap \operatorname{succ}_{p_{B}}(s) \neq \emptyset$. Let $\ell \in f^{-1}(k) \cap \operatorname{succ}_{p_{B}}(s)$. Thus $s \frown \ell$ favors $k \in \dot{A}_{n}$. Hence there is $q \leq p_{B}$ whose stem extends $s \frown \ell$ such that $q \Vdash k \in \dot{A}_{n}$, again a contradiction.

$2 \Rightarrow 3$ is trivial.

$(3 \Rightarrow 1$.) Assume that there is a countably tall ideal $\mathcal{J}$ Katětov below $\mathcal{K} \uparrow X$, for some $X \in \mathcal{K}^{+}$, witnessed by a function $f$. Let $\dot{Y}$ be a name for 
the range of the $\mathbb{L}(\mathcal{G})$-generic function. Then $\Vdash_{\mathbb{L}(\mathcal{G})} f[\dot{Y} \cap X]$ is infinite and, moreover, $\Vdash_{\mathbb{L}(\mathcal{G})} f[\dot{Y} \cap X]$ is almost disjoint from every element of $\mathcal{J}$. Hence $\vdash_{\mathbb{L}(\mathcal{G})} \mathcal{J}$ is not tall and therefore not countably tall.

It is an immediate corollary of the proposition that $\mathbb{L}(\mathcal{H})$ strongly preserves countable tallness if $\mathcal{K}=\mathcal{H}^{*}$ is a Fréchet ideal, as every Fréchet ideal obviously satisfies condition (1).

Lemma 5. Finite support iteration of forcings strongly preserving countable tallness strongly preserves countable tallness.

Proof. This is a standard argument. We provide the details for the sake of completeness. Obviously, it suffices to consider limit stages of cofinality $\omega$.

Let $\left(\mathbb{P}_{k}, \dot{\mathbb{Q}}_{k}: k \in \omega\right)$ be a finite support iteration of ccc forcing such that each $\mathbb{P}_{k}$ strongly preserves countable tallness.

Let $\left(\dot{A}_{n}: n \in \omega\right)$ be a sequence of $\mathbb{P}_{\omega}$-names for infinite subsets of $\omega$. In the intermediate extension $V\left[G_{k}\right]$ find a decreasing sequence of conditions $\left(p_{n, k}: n \in \omega\right)$ and infinite subsets $A_{n, k}$ of $\omega$ such that

$$
p_{n, k} \Vdash_{\mathbb{P}_{[k, \omega)}} \text { "the first } n \text { elements of } A_{m, k} \text { and } \dot{A}_{m} \text { agree for } m \leq n \text { ". }
$$

The $A_{n, k}$ are approximations to $\dot{A}_{n}$.

Now, as each $\mathbb{P}_{k}$ strongly preserves countable tallness, there are infinite subsets $B_{n, k}$ of $\omega$ such that for every $B \in[\omega]^{\omega}$, if $B \cap B_{n, k}$ is infinite for all $n$ then

$$
\Vdash_{\mathbb{P}_{k}} B \cap \dot{A}_{n, k} \text { is infinite for all } n \text {. }
$$

Consider $\left\{B_{n, k}: n, k \in \omega\right\}$ and let $B \in[\omega]^{\omega}$ be such that $B \cap B_{n, k}$ is infinite for all $n$ and $k$. To finish the proof, it suffices to show that $\Vdash_{\mathbb{P}_{\omega}} B \cap \dot{A}_{n}$ is infinite for all $n$.

If not, then there are a $q \in \mathbb{P}_{\omega}, n \in \omega$ and $m \in \omega$ such that $q \Vdash_{\mathbb{P}_{\omega}}$ $B \cap \dot{A}_{n} \subseteq m$. Let $k$ be such that $q \in \mathbb{P}_{k}$.

Let $G_{k}$ be a $\mathbb{P}_{k}$-generic such that $q \in G_{k}$. As $B \cap A_{n, k}$ is infinite, let $\ell \geq m$ with $\ell \in B \cap A_{n, k}$. For large enough $m$,

$$
p_{m, k} \Vdash_{\mathbb{P}_{[k, \omega)}} \ell \in \dot{A}_{n} .
$$

Since $q \in G_{k}$, this contradicts the initial assumption about $q$.

The proof of Theorem 2 is now immediate. By taking care of all $\omega_{1}$-generated ideals $\mathcal{I}$ via book-keeping, we iterate forcing notions of the type $\mathbb{L}(\mathcal{G})$ for $\omega_{2}$ steps with finite support. By Lemmata 3 and 4 , we add $X \in\left(\mathcal{I}^{<\omega}\right)^{+}$such that $\mathcal{I}^{<\omega} \mid X$ is countably tall (and so $\mathcal{I}^{<\omega}$ is not Fréchet). By Lemmata 2, 5 and Proposition 1, the countable tallness of $\mathcal{I}^{<\omega} \uparrow X$ is preserved along the iteration, and we are done.

\section{Final remarks and questions}

Obviously, the question of Gruenhage and Szeptycki remains open. Even though, we do not know whether in the model of ZFC just constructed 
there are any $F U_{f i n}$-filters of uncountable character (necessarily of character $\mathfrak{c}=\aleph_{2}$ ).

It should also be noted that there are (consistently) topologies on $\left([\omega]^{<\omega}, \triangle\right)$ which are not of the form $\tau_{\mathcal{F}}$, yet make $\left([\omega]^{<\omega}, \triangle\right)$ a nonmetrizable Fréchet-Urysohn group. An easy example can be described as follows:

Let $X \subseteq \mathcal{P}(\omega)$ be such that $X$ separates points of $[\omega]^{<\omega}$, i.e. for every $a \in[\omega]^{<\omega} \backslash\{\emptyset\}$ there is an $x \in X$ such that $|a \backslash x|$ is odd. Let

$$
\mathcal{F}_{X}=\left\{A \subseteq[\omega]^{<\omega}:\left(\exists F \in[X]^{<\omega}\right)(\forall a \in A)(\forall x \in F) \quad|a \backslash x| \text { is even }\right\} .
$$

By declaring $\mathcal{F}_{X}$ the neighbourhood base at $\emptyset$, we introduce a Hausdorff group topology $\tau_{X}$ on $G$. To see this, consider the function $\varphi:[\omega]^{<\omega} \longrightarrow$ $2^{X}$ defined by $\varphi(a)(x)=0$ if and only if $|a \backslash x|$ is even. Then $\varphi$ is a group homomorphism and as $X$ separates points of $[\omega]^{<\omega}$, it is an embedding. It is easily seen that the topology $\tau_{X}$ is just the subspace topology induced by $\varphi$ (viewing $[\omega]^{<\omega}$ as a subgroup of $2^{X}$ ).

Now, it is easy to verify that if $X$ is a $\gamma$-set then the topology $\tau_{X}$ on $\left([\omega]^{<\omega}, \triangle\right)$ is Fréchet-Urysohn. Indeed, let for $a \in[\omega]^{<\omega}$

$$
U_{a}=\{x \in \mathcal{P}(\omega):|a \backslash x| \text { is even }\}
$$

and for $A \subseteq[\omega]^{<\omega}$ let $\mathcal{U}_{A}=\left\{U_{a}: a \in A\right\}$. Note that $U_{a}$ is a clopen subset of $\mathcal{P}(\omega)$ for every $a \in[\omega]^{<\omega}$. It is now immediate from the definition of $\mathcal{F}_{X}$ that the topology $\tau_{X}$ is Fréchet-Urysohn at 0 (and hence FréchetUrysohn) if and only if for every infinite $A \subseteq[\omega]^{<\omega}$ if $\mathcal{U}_{A}$ is an $\omega$-cover of $X$ then there is an infinite $B \subseteq A$ such that $\mathcal{U}_{B}$ is a $\gamma$-cover of $X$ (see either of [GN, GS1, Ny2] for the definitions of $\gamma$-sets and corresponding covers).

The fundamental difference between the topologies of the type $\tau_{\mathcal{F}}$ and $\tau_{X}$ is that the group topology $\tau_{X}$ is always pre-compact, i.e. has a group compactification, while the topology $\tau_{\mathcal{F}}$ is pre-compact only if $\mathcal{F}$ is the filter of co-finite sets. We do not know, whether there is a non-metrizable topology of the type $\tau_{X}$ in our model. More about pre-compact group topologies on countable abelian groups will appear in $[\mathrm{HR}]$.

Acknowledgements. M. Hrušák would like to thank Ulises Ariet Ramos-García for careful reading of the paper and fruitful discussions on the subject. J. Brendle would like to thank Dmitri Shakhmatov for stimulating discussions on the topic of this paper during his visit to Ehime University in February 2008. He is also grateful to the second author and the Instituto de Matemáticas in Morelia for support and for having provided a motivating research environment during his visit there in February 2006 when most of this research was carried out.

\section{References}

[Ar] A. V. Arhangel'skii, Classes of topological groups, Russian Math Surveys 36 (1981), 151-174.

[Br1] J. Brendle, Van Douwen's diagram for dense sets of rationals, Ann. Pure Appl. Logic 143 (2006), 54-69. 
[Br2] J. Brendle, Independence for distributivity numbers, in: Algebra, Logic, Set Theory. Festschrift für Ulrich Felgner zum 65. Geburtstag (B. Löwe, ed.), Studies in Logic Vol. 4, College Publications, London, 2007, 63-84.

[Do] A. Dow, Two classes of Fréchet-Urysohn spaces, Proc. Amer. Math. Soc. 108 (1990), 241-247.

[GS1] G. Gruenhage, P.J. Szeptycki, Fréchet-Urysohn for finite sets, Top. Appl. 151 (2005), 238-259.

[GS2] G. Gruenhage, P.J. Szeptycki, Fréchet-Urysohn for finite sets, II, Top. Appl. 154 (2007), 2856-2872.

[GN] J. Gerlits, Zs. Nagy, Some properties of $C(X)$, I, Top. Appl. 14 (1982), 151-161.

[HG] M. Hrušák, S. García-Ferreira, Ordering MAD families a la Katětov, J. Symb. Logic 68 (2003), 1337-1353.

[HR] M. Hrušák, U. A. Ramos-García, Pre-compact topologies on countable abelian groups, in preparation (2008).

[HZ] M. Hrušák, J. Zapletal, Forcing with quotients, Arch. Math. Logic 47 (2008), 719-739.

[MT] J. T. Moore, S. Todorčević, The metrization problem for Fréchet groups, in: Open Problems in Topology II (E. Pearl, ed.), Elsevier, 2007, 201-206.

[Ny1] P. Nyikos, Subsets of ${ }^{\omega} \omega$ and the Fréchet-Urysohn and $\alpha_{i}$ properties, Top. Appl. 48 (1992), 91-116.

[Ny2] P. Nyikos, The Cantor tree and the Fréchet-Urysohn property, Ann. New York Acad. Sci 552 (1989), 109-123.

[RS] E. Reznichenko, O. Sipacheva, Fréchet-Urysohn type properties in topological spaces, groups and locally convex vector spaces, Moscow Univ. Math. Bull 54 (1999), 33-38.

[Si] O. Sipacheva, Spaces Fréchet-Urysohn with respect to families of subsets, Top. Appl. 121 (2002), 305-317. 